\title{
Reflexões sobre o Cuidado ao Idoso Hospitalizado através da Biblioterapia: Um Método Terapêutico Humanizado nos Hospitais
}

\author{
Juliana Maria Silva Bernardo ${ }^{\text {; }}$ Claudia Daniele Barros Leite Salgueiro ${ }^{2}$. \\ Paula Layse da Silva ${ }^{1}$; Jaqueline Cordeiro Lopes ${ }^{1}$; Aline Bezerra Sobrinho ${ }^{1}$;
}

Resumo: Trata-se de uma revisão integrativa sobre a produção científica acerca da biblioterapia aplicada aos idosos em hospitalização. Objetivo: O estudo buscou evidenciar o perfil dos trabalhos publicados em âmbito nacional, no período de 2007 a 2015, com o levantamento feito a partir do Portal Regional da Biblioteca Virtual de Saúde (BVS), direcionando para periódicos indexados nas bases Scientific Electronic Library Online (SCIELO), Literatura Latino Americana e do Caribe em Ciências da Saúde (LILACS), Sistema Online de Busca e Análise de Literatura Médica (MEDLINE) e Base de Dados de Enfermagem (BDENF). Método: A amostra foi apresentada em dois quadros sinóticos com a distribuição dos artigos analisados. Conclusão: Os dados evidenciaram que a biblioterapia ainda é um assunto escasso em estudos e nas graduações. Também é uma prática terapêutica pouco aplicada no âmbito hospitalar, e quando é realizada, em sua maioria, é voltada para o público infantil, sendo muito raramente abordada com pacientes idosos. Tais fatos podem indicar um panorama de pouca divulgação de métodos que visam humanizar o cuidado.

Descritores: Serviços de saúde para idosos; biblioterapia; humanização da assistência.

\section{Reflections on the Care of the Hospitalized Elderly Through Bibliotherapy: A Humanized Therapeutic Method in Hospitals}

\begin{abstract}
This is an integrative review of the scientific literature about the bibliotherapy applied to hospitalized elderly. Objective: The study aimed to highlight the profile of the works published in the national context, from 2007 to 2015, with the survey made from the Regional Portal of the Virtual Health Library (VH), targeting journals indexed in the Scientific Electronic Library Online (SCIELO), Latin American and Caribbean Literature in Health Sciences (LILACS), Online System of Search and Analysis of Medical Literature (MEDLINE) and Nursing Database (BDENF). Method: The sample was presented in two synoptic tables with the distribution of the articles analyzed. Conclusion: The data showed that bibliotherapy is still a scarce subject in studies and graduations. It is also a poorly applied therapeutic practice in the hospital setting, and when it is performed, it is mostly aimed at the infantile public, being very rarely approached with elderly patients. Such facts may indicate a lack of dissemination of methods that seek to humanize care.
\end{abstract}

Keywords: Keywords: Health services for the elderly; bibliotherapy; humanization of care.

\footnotetext{
${ }^{1}$ Discentes do curso de bacharelado em enfermagem - Instituto Federal de Educação, Ciência e Tecnologia de Pernambuco (IFPE) - Campus Pesqueira.

${ }^{2}$ Doutora em Psicologia Clínica pela Universidade Católica de Pernambuco (UNICAP), Mestra em Ciências da Saúde pela Universidade de Pernambuco (UPE), Pós Graduada em Saúde Coletiva pela mesma Universidade. Psicóloga formada pela Universidade Católica de Pernambuco (UNICAP). Docente do curso Bacharelado em Enfermagem do Instituto Federal de Educação, Ciência e Tecnologia de Pernambuco (IFPE) - Campus Pesqueira. Contato: claudia.leite@ pesqueira.ifpe.edu.br.
} 


\section{Introdução}

O envelhecimento populacional é um fato incontestável no cenário atual. O Brasil, segundo dados prorrogativos, será o sexto país com maior índice de população idosa em todo mundo, até 2025. Essa mudança de panorama acarreta muitas implicações econômicas, sociais, e em saúde. Os idosos tendem a consumir em maior escala os serviços de saúde, bem como sua permanência em internamentos é mais elevada (CASTRO et al., 2013).

Considerando-se a entrada e permanência do idoso no ambiente hospitalar devido a questões de vulnerabilidade da saúde, torna-se imperioso considerar que a permanência em ambiente hospitalar pode incidir em fatores estressores que podem influir diretamente em sua patologia, de forma negativa (BENEDETTI, 2008). Muitos já se encontram fragilizados e, ao deparar-se com o diagnóstico, tratamento, quebra da rotina, ambiente e pessoas que não atuam em seu cotidiano, tendem a associar o ambiente hospitalar, e sua equipe, como um local estressor e extremamente centrado em sua patologia (MELO, 2013).

No modelo de saúde vigente, o paciente é visto como a patologia que o acomete, o que causa certo distanciamento entre paciente e profissional de saúde, já que nenhum elo afetivo é criado. Em consequência disso, a permanência do idoso nesse ambiente hospitalar tão mecanizado, gera quadros de ansiedade e estresses profundos (MELO, 2013; CERIBELLI et al., 2009).

Pensando em, gradativamente, mudar o cenário desse modelo mecanicista, foi criada a Política de Humanização da Assistência à Saúde, que tem como característica humanizar as práticas e cuidados de saúde, melhorando, sobretudo, a qualidade de atenção que é dispensada ao paciente (BENEDETTI, 2008). O paciente deixa de ser visto como um corpo doente, e passa a ser observado de forma holística. Com essa pequena e tão significativa mudança, o elo entre paciente e profissional em saúde é fortalecido, o que pode influir muito na qualidade de vida desse paciente durante o internamento (CERIBELLI et al, 2009).

O ambiente hospitalar é composto de uma equipe multidisciplinar, e toda ela deve estar pautada na política de humanização. No entanto, há um profissional em especial que passa maior parte do seu tempo ao lado do paciente, sendo assim extremamente importante a criação 
de uma comunicação efetiva e o fortalecimento de um elo (SILVA; SOUZA; ANDRADE, 2015).

De tal modo, o profissional de enfermagem precisa buscar essa aproximação com seu paciente, visando adotar medidas que lhes proporcione mais conforto e bem-estar, e é exatamente nesse âmbito que as práticas terapêuticas ganham espaço (MELO, 2013; CERIBELLI, et al 2009; SILVA; SOUZA; ANDRADE, 2015).

Práticas terapêuticas leves são adotadas cada vez com mais frequência nas clínicas hospitalares, o que demonstra essa busca, cada vez mais ativa, por parte dos profissionais, por formas de minimizar o ambiente estressor e fortalecer vínculos com seus pacientes. O processo é gradual, mas é notado com cada vez mais frequência (FONSECA; GEOVANINI, 2013).

A ludicoterapia é uma prática terapêutica muito utilizada nas clínicas pediátricas, e a exemplo disso temos a musicoterapia e biblioterapia, usada nas clínicas para adultos (CERIBELLI et al, 2009).

Neste cerne, a biblioterapia tem se destacado como forte método calmante, mediador de comunicação e de fortalecimento do vínculo paciente/profissional. Esta, por sua vez, é apontada como uma maneira de humanizar o atendimento, além de todo o processo terapêutico que a envolve (MELO, 2013).

Os dados acima supõem que, através do método, questões como, fortalecimento da comunicação profissionais/paciente/família, exposição de sentimentos, possiblidade de ressignificação de situações, melhora na qualidade de vida e estimulação, podem possibilitar ao paciente idoso a efetivação de alta médica e retorno ao seu lar e às suas atividades de rotina.

$\mathrm{O}$ enfermeiro é apontado como profissional da equipe que mais orbita em volta do paciente, evidenciando assim a necessidade de uma interação mais profunda com seu paciente (CERIBELLI et al, 2009). O desenvolvimento tecnológico tem contribuído para o trabalho desses profissionais, no entanto tem cooperado para o reforço do atendimento biomédico (CASTRO et al, 2013).

Quanto à temática, também é importante destacar acerca da vinculação. A dificuldade de formação de vínculo traz consigo um problema para a implementação da atenção à saúde humanizada. Aquela que dá ênfase no paciente como um ser biopsicossocial, que necessita de um olhar holístico, principalmente voltado da equipe de enfermagem (SILVA; SOUZA; ANDRADE, 2015). 
Para os enfermeiros, é importante conseguir angariar subsídios que auxiliem na qualidade da assistência prestada. Buscar o bem-estar físico e mental do seu paciente é a melhor forma de humanizar o atendimento, atrelando cuidares que são pilares para a assistência holística (CERIBELLI et al, 2009).

Dentro desses subsídios, as práticas terapêuticas ganham força nesse meio, aparecendo como métodos humanizados que contribuem como mediador de comunicação, formador de vínculos e forte aliado no alivio do sofrimento psíquico causado pela hospitalização (MELO, 2013).

Relacionando-se as temáticas, é imperioso considerar que a biblioterapia é um método terapêutico que vem sendo utilizado nas clínicas hospitalares, entretanto, atingindo em maioria, crianças hospitalizadas.

Considerando-se a potencialização do processo de hospitalização para a fragilização do paciente, e quando se trata de um público já fragilizado, como os idosos, destaca-se que devese escolher o elemento que tenha maior poder terapêutico com tal público (MELO, 2013; FONSECA; GEOVANINI, 2013; JERÔNIMO et al, 2012).

A palavra biblioterapia, vem do grego, e é composta por dois termos "biblion" (material bibliográfico para leitura) e "therapein" (tratamento ou terapia), essa junção confere à biblioterapia o significado de tratamento através da leitura (SILVA, 2013).

Indubitavelmente, considera-se que a leitura entra nesse âmbito enquanto atividade de lazer, humanizando o processo de hospitalização, por meio da leitura dirigida, ou biblioterapia (Almeida, 2011). As atividades lúdicas somadas à leitura, viabilizam o desenvolvimento de um processo que facilita a interação de valores, ações e sentimentos relacionados ao desenvolvimento pessoal, favorecendo dessa maneira, diversos benefícios ao paciente, pois, a leitura faz com que o indivíduo adquira novos conhecimentos, novas percepções e tenha uma nova visão do mundo (VALÊNCIA; MAGALHÃES, 2015).

Em suma, a linguagem em movimento, a conversa, a troca de conhecimentos e a identificação com o personagem são apontados como alicerces dessa prática terapêutica. Assim como seu nome evidencia, uma terapia por meio dos livros (JERÔNIMO et al, 2012).

A leitura ainda é apontada como ativadora de sentimentos catárticos. A palavra catarse é definida na psicanálise como liberação de pensamentos e emoções que estavam reprimidas no inconsciente, seguindo-se de alívio emocional. Já em termos médicos é apontada como 
psicoterapia que visa à remissão dos sintomas através da exteriorização verbal e emocional de afetos reprimidos (BENEDETTI, 2008; JERÔNIMO et al, 2012).

Essa catarse é o ponto chave no tratamento terapêutico oferecido pela biblioterapia. Isso porque, essa liberação de emoções reprimidas pode aliviar tensões que podem estar contribuindo para o adoecimento mental e até físico desse idoso (MELO, 2013).

As atividades lúdicas somadas à leitura, viabilizam o desenvolvimento de um processo que facilita a interação de valores, ações e sentimentos, relacionados ao desenvolvimento pessoal, favorecendo dessa maneira diversos benefícios ao pacientem, pois, a leitura faz com que o indivíduo adquira novos conhecimentos, percepções e tenha uma nova visão do mundo (VALÊNCIA; MAGALHÃES, 2015).

Aristóteles há muitos anos atrás já observava a pacificação das emoções proporcionada pela terapia através da identificação com o personagem narrado. A catarse significa purificação, e acontece quando sentimentos conflituosos são trazidos do subconsciente e superados, a partir da leitura (MELO, 2013; CERIBELLI et al, 2009).

Nessa ação, além do processo catártico, ainda é possível notar o insight, que é o momento de reflexão sobre os sentimentos e a possibilidade de exteriorizá-los e trabalhá-los. São esses dois componentes que precisam ser analisados pelo mediador da leitura, pois é válido lembrar que a biblioterapia tem fins terapêuticos e não apenas recreativos (MELO, 2013; JERÔNIMO et al, 2012).

Assim, o profissional de enfermagem ou qualquer outro profissional de saúde que busca implementar esse método em sua clínica, necessitará de capacitação. Bem como, capacitar os elementos daquela equipe para lidar com os pormenores somatórios da prática (CERIBELLI et al, 2009).

A capacitação é importante para que esses profissionais saibam buscar um modo de chegar ao seu objetivo por meio da biblioterapia. Isso se deve ao fato de que o método biblioterapêutico não se resume a ler uma história, esta pode ser dramatizada, narrada, tudo depende do nível cultura, interesse ou mesmo algum bloqueio desse paciente, como uma dificuldade auditiva, por exemplo (BENEDETTI, 2008; SILVA; SOUZA; ANDRADE, 2015).

Portanto, o perfil do paciente deve ser traçado anteriormente pelo mediador, em seguida à busca ativa por histórias que possam trazer para a superfície sentimentos catárticos nesse paciente. Por isso é importante não apenas a capacitação, mas também um trabalho 
multidisciplinar nesse âmbito, com a ajuda de psicólogo, assistente social, e um profissional em biblioteconomia, por exemplo (CERIBELLI et al, 2009; ALMEIDA, 2011).

Acredita-se que ficarão evidentes os prós advindos através da biblioterapia, sejam eles através da liberação de emoções, identificação com os personagens, aproximação com o enfermeiro ou mesmo prática de lazer para auxiliar no alívio do estresse causado pelo ambiente hospitalar, ainda há um resgate emocional e de tradicionalismo vivido por esse idoso, que se sente perdido em meio à tecnologia, e o livro traz esse resgate do passado (MELO, 2013; JERÔNIMO et al, 2012).

O idoso, assim como qualquer pessoa submetida ao internamento hospitalar, tem sua rotina quebrada, ele afasta-se pessoas do seu convívio ou mesmo as atividades são reprimidas, o que remete o hospital a um ambiente de sofrimento (MELO, 2013). Além da imediação da morte que o ambiente carrega, o que certamente pode trazer angústia e medo (FONSECA; GEOVANINI, 2013).

Essa carência, normalmente conjunta à idade avançada, ratifica a necessidade de uma prestação de assistência humanizada, e torna notória a necessidade de elementos que tornem o processo de internação menos traumatizante e mais acolhedor. Onde, nesse campo, a biblioterapia tem muito a contribuir (BENEDETTI, 2008; MELO, 2013; CERIBELLI et al, 2009). Uma vez que é importante compreender as situações emocionais, psicológicas e sentimentais, quais suas expectativas, desejos, sonhos, desejos de mudanças e o que acontecem em relação a seus sentimentos momentâneos, assim como também é importante saber quais são suas queixas sobre o que lhe faz mal (SILVA, 2013).

A biblioterapia é uma maneira de leitura e dialogo grupal que viabiliza interpelação entre as pessoas, favorecendo que os indivíduos expressem seus sentimentos. Dessa maneira, os pacientes podem partilhar seus sentimentos com os enfermeiros, os psicólogos ou outros profissionais de saúde e também, com outros pacientes, buscando assim que exista a resolução de seus problemas (VALÊNCIA; MAGALHÃES, 2015).

A leitura é, sem dúvida, uma atividade inteiramente benéfica, recomendada para diversos somatórios no processo de humanização da hospitalização. É pautada em um processo de cura através das letras e proporciona ao idoso hospitalizado, além de outras coisas, distrairse, sonhar, rir, imaginar. Fortalecendo vínculos, mediando comunicação e trabalhando para um novo olhar ao ambiente hospitalar. 
Neste sentido, presente estudo teve como objetivo, explorar o conhecimento e as práticas acerca da biblioterapia, assim como também, objetivou fortalecer a visão do método biblioterapêutico como prática humanizada para pacientes idosos, internados em clínicas hospitalares, uma vez que esse método de tratamento secundário, auxilia no tratamento medicamentoso do paciente em questão, buscando consequentemente, reduzir os seus dias de internamento, o tornar melhor e com mais qualidade de vida.

\section{Metodologia}

Trata-se de um estudo de revisão integrativa. Esta categoria de pesquisa possibilita a análise de pesquisas e a síntese dos conceitos de forma ampla, visando a necessidade do conhecimento científico para preparação e desenvolvimento do artigo. Algumas etapas devem ser seguidas para elaboração da revisão integrativa, são essas: definição da pergunta/problemática; coleta bibliográfica, classificação dos dados, discussão dos estudos incluídos, e resultados finais (CROSSETTI, 2012).

Através da revisão integrativa, a pesquisa científica na área da saúde, notadamente no campo da enfermagem, ganha uma metodologia significativa para a aplicabilidade da Prática Baseada em Evidência (PBE) no contexto social. Esta se distingue das demais metodologias, por ser uma ferramenta articulada e subsidiada em fontes confiáveis (SOUZA; SILVA E CARVALHO, 2010). No caso do presente artigo, fontes brasileiras correspondentes ao tema central.

A escolha por utilizar a integração de dados se justifica mediante a evidência possibilitada por esse método, que permite juntar estudos experimentais e não-experimentais. Originando um maior entendimento acerca do fenômeno estudado, o que a torna a mais ampla abordagem metodológica em relação às demais revisões (SOARES et al, 2014).

Como primeira etapa foi formulada a seguinte pergunta condutora: "Como a biblioterapia enquanto prática terapêutica humanizada pode auxiliar idosos internados em âmbito hospitalar?'

Na segunda etapa realizou-se a busca dos artigos publicados acerca da temática em abril de 2017, nas bases de dados Biblioteca Virtual em Saúde (BVS), através das bases de dados Scientific Electronic Library Online (SCIELO), Literatura Latino Americana e do Caribe em Ciências da Saúde (LILACS), Sistema Online de Busca e Análise de Literatura Médica 
(MEDLINE) e Base de Dados de Enfermagem (BDENF). Para a busca dos artigos, foram utilizados os Descritores em Ciências da Saúde (DeCS) Serviços de saúde para idosos, Biblioterapia”, Humanização da assistência. Foram combinados de formas distintas, como através da utilização de sinônimos, assim como do operador booleano "AND”. Como pode ser observado no fluxograma contido na figura 1.

As bases de dados supramencionadas foram eleitas devido ao quantitativo de indexação de artigos da área da saúde, ainda, por contemplarem estudos primários, assim como também, devido à indexação de artigos nas temáticas relacionadas a área de saúde, especialmente à enfermagem. Os descritores foram combinados de diferentes formas com o objetivo de expandir a busca pelos estudos. Foram consideradas as variações terminológicas, bem como sinônimos. No intuito de uma busca sensibilizada, foram utilizados os operadores booleanos AND para ocorrência simultânea de assuntos.

Os operadores booleanos são expressões utilizadas para fazer associações de palavras durante uma pesquisa, os mais utilizados são: AND/ OR/ AND NOT. São aceitas a combinação de duas ou mais palavras/assuntos no(s) campo(s) de busca, além disso, pode-se aperfeiçoar a pesquisa alterando os operadores ou acrescentando-se descritores (BIREME, 2009).

$\mathrm{Na}$ terceira etapa, os estudos foram selecionados de acordo com critérios de inclusão e exclusão. Elencados como critérios de inclusão: Artigos publicados em português, com os resumos disponíveis nas bases de dados selecionadas, no período compreendido entre 2007-2015. Quanto ao corte do período estudado, o mesmo justificou-se por afirmar a contemporaneidade dos dados, dando enfoque as tendências das investigações analisadas. 
Figura 1: Fluxograma das etapas da coleta dos artigos nas bases de dados.

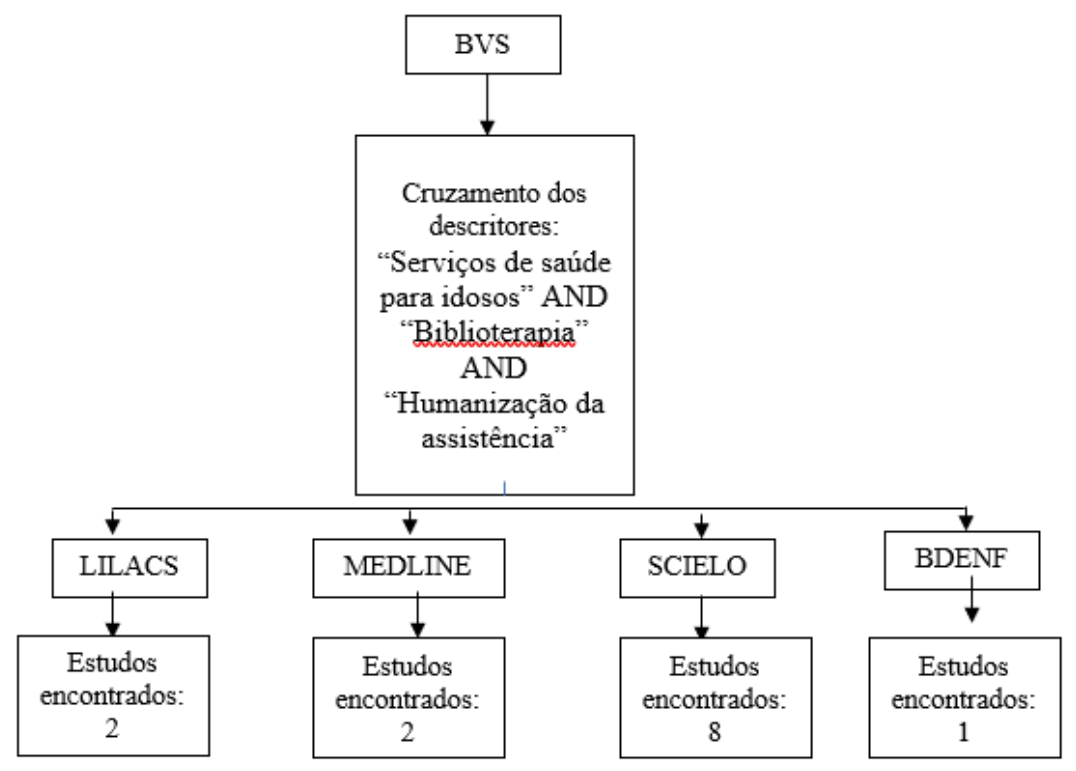

Fonte: Dados levantados pelos autores (Pesqueira, 2018).

Através dos descritores, foram encontrados inicialmente 166 artigos, após a leitura dos resumos e aplicação dos critérios de inclusão e exclusão, 4 foram selecionados para subsidiar os resultados da pesquisa e possibilitar posterior catalogação no quadro.

Posteriormente teve início a leitura e interpretação dos dados, bem como a categorização dos artigos, por sua relevância. Em seguida, foram elencados os níveis de evidência. O nível de evidência científica é uma variável de suma importância, uma vez que visa sintetizar determinado conteúdo, contendo resultados significativos nas atividades assistenciais, e na prática baseada em evidências (SOUZA; SILVA E CARVALHO, 2010).

Os níveis de evidências são caracterizados de forma metodológica da seguinte maneira: Nível 1: evidências a partir da meta-análise de múltiplos estudos clínicos controlados e randomizados; Nível 2: evidências obtidas em estudos individuais com delineamento experimental; Nível 3: evidências de estudos quase-experimentais; Nível 4: evidências de estudos descritivos (não-experimentais) ou com abordagem qualitativa; Nível 5: evidências provenientes de relatos de caso ou de experiência; Nível 6: evidências baseadas em opiniões de especialistas (SOUZA, SILVA E CARVALHO, 2010). 
Na quinta etapa, os dados foram expostos nos resultados e discussão, e por fim na sexta, foram elaborados quadros sinóticos através do Microsoft Word 2010, possibilitando ao leitor a avaliação da aplicabilidade da revisão integrativa elaborada, de forma a atingir o objetivo desse método, ou seja, impactar positivamente no uso de tecnologias leves de cuidado, na assistência de enfermagem com idosos internados em clínicas hospitalares.

Os quadros construídos contêm as seguintes informações: código do artigo, base de dados, periódico, região onde a pesquisa foi realizada, ano de publicação, autor (es), título, objetivos, principais contribuições, área de publicação, tipo da pesquisa e nível de evidência.

\section{Resultados e Discussão}

A bibliografia que compôs o quadro tornou possível a análise das inúmeras aplicabilidades da biblioterapia como tratamento terapêutico humanizado. Evidenciando as positividades quanto à prática terapêuticas e dando embasamento para futuros profissionais da área da enfermagem a usar como elemento de humanização da assistência de saúde (MELO, 2013).

O presente estudo deu ênfase à biblioterapia voltada para o público idoso em processo de internação, o que não queira dizer que o método não possa ou não deva ser usado com outros públicos. Também houve destaque quanto ao profissional de enfermagem na aplicação da biblioterapia, no entanto, desde que haja capacitação adequada, o método terapêutico pode ser adotado por outros profissionais da área de saúde (BENEDETTI, 2008; JERÔNIMO et al, 2012).

A enfermagem precisa atrelar os cuidados biológicos e da mente para que haja um melhoramento completo do ser. Observando que o físico e psicológico estão fortemente atrelados, portanto para um atendimento humanizado e holístico há necessidade de cuidar do ser em seu todo (SILVA; SOUZA; ANDRADE, 2015). 
Quadro 01 - Classificação dos artigos quanto ao objetivo geral dos estudos, assim como, suas principais contribuições em publicações entre 2008-2015.

\begin{tabular}{|c|c|c|c|c|}
\hline CD & Autor(es) & Título & Objetivo & Principais Contribuições \\
\hline$A 1$ & $\begin{array}{lr}\text { Vivian Carla de Castro; } \\
\text { Ana Carla } & \text { Borghi; } \\
\text { Pâmela } & \text { Patrícia } \\
\text { Mariano; } & \text { Carlos } \\
\text { Alexandre } & \text { Molena } \\
\text { Fernandes; Thaís Aidar } \\
\text { de Freitas Mathias; } \\
\text { Lígia Carreira. }\end{array}$ & $\begin{array}{lr}\text { Perfil } & \text { de } \\
\text { internações } & \\
\text { hospitalares } & \text { de } \\
\text { idosos } & \text { no } \\
\text { âmbito } & \text { do } \\
\text { Sistema } & \text { Único } \\
\text { de Saúde } & \end{array}$ & $\begin{array}{l}\text { Caracterizar o perfil } \\
\text { das internações } \\
\text { hospitalares das } \\
\text { Regionais de Saúde } \\
\text { do Paraná, Brasil, nos } \\
\text { anos de } 2008 \text { a } 2011 . \\
\text { Traçar um perfil geral } \\
\text { a respeito dessas } \\
\text { internações. }\end{array}$ & $\begin{array}{l}\text { Evidenciou o perfil das } \\
\text { internações hospitalares de } \\
\text { idosos nos permite vivenciar o } \\
\text { momento demográfico de } \\
\text { determinada região. } \\
\text { Evidenciou também os tipos de } \\
\text { patologias e quais atendimentos } \\
\text { devem ser prestados, abrindo } \\
\text { caminhos para adoção de } \\
\text { elementos que auxiliem os } \\
\text { profissionais. }\end{array}$ \\
\hline$A 2$ & $\begin{array}{lr}\text { Carina } & \text { Ceribelli; } \\
\text { Lucila } & \text { Castanheira } \\
\text { Nascimento; } & \text { Soraya } \\
\text { Maria } & \text { Romano } \\
\text { Pacífico; } & \text { Regina } \\
\text { Aparecida } & \text { Garcia de } \\
\text { Lima } & \end{array}$ & $\begin{array}{l}\text { A mediação da } \\
\text { leitura como } \\
\text { recurso de } \\
\text { comunicação } \\
\text { com crianças } \\
\text { hospitalizadas. }\end{array}$ & $\begin{array}{l}\text { Entender como a } \\
\text { comunicação com o } \\
\text { paciente hospitalizado } \\
\text { é fator imperioso para } \\
\text { uma prática } \\
\text { humanística. } \\
\text { Utilizando-se como } \\
\text { mediador a leitura. }\end{array}$ & $\begin{array}{l}\text { Mostrou que a assistência } \\
\text { prestada em ambiente hospitalar } \\
\text { pela equipe de enfermagem deve } \\
\text { ser pautada em um olhar } \\
\text { holístico, usando como base, a } \\
\text { adoção de elementos que } \\
\text { facilitem a formação de vínculos } \\
\text { com seu paciente. A adoção da } \\
\text { biblioterapia, por exemplo, pode } \\
\text { garantir maior aproximação e } \\
\text { um entendimento mais facilitado } \\
\text { com os anseios que cercam } \\
\text { aquele paciente. }\end{array}$ \\
\hline$A 3$ & $\begin{array}{l}\text { Silva, A.P.S; Souza, B. } \\
\text { O.R; Andrade E.R. }\end{array}$ & $\begin{array}{l}\text { Enfermagem e } \\
\text { psicologia: } \\
\text { Parceria na arte } \\
\text { do cuidar } \\
\text { profissional }\end{array}$ & $\begin{array}{ll}\text { Refletir sobre } & \text { a } \\
\text { relação entre } & \text { a } \\
\text { enfermagem } & \text { e } \\
\text { psicologia na prática } \\
\text { humanizada. }\end{array}$ & $\begin{array}{l}\text { Demonstrou a psicologia atual } \\
\text { como ferramenta crucial para } \\
\text { que uma assistência de } \\
\text { enfermagem possa ocorrer. }\end{array}$ \\
\hline A4 & $\begin{array}{l}\text { Anelise Fonseca; } \\
\text { Fátima Geovanini }\end{array}$ & $\begin{array}{l}\text { O uso da } \\
\text { literatura } \\
\text { infantil na UTI: } \\
\text { Um convite à } \\
\text { reflexão e à } \\
\text { discussão para } \\
\text { os profissionais } \\
\text { sobre a morte e } \\
\text { o morrer. }\end{array}$ & 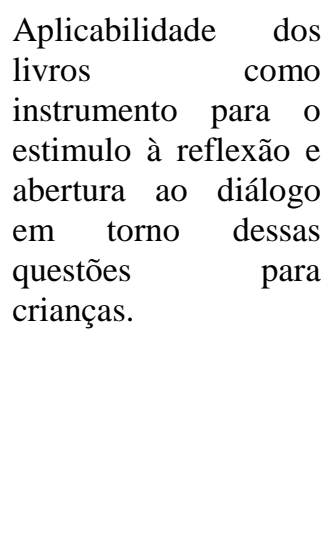 & $\begin{array}{l}\text { Demonstrou temas como dor, } \\
\text { adoecimento, perda, processo de } \\
\text { morrer. Morte e luto precisam } \\
\text { ser tratados de forma realista, } \\
\text { reconhecendo-os como comuns } \\
\text { ao ciclo da vida. } \\
\text { Demonstrou que a morte ainda é } \\
\text { considerada como um tabu. Os } \\
\text { profissionais que lidam } \\
\text { diariamente com isso precisam } \\
\text { tentar elencar formas de } \\
\text { amenizar o fator estressor que é } \\
\text { a morte iminente. }\end{array}$ \\
\hline
\end{tabular}

Fonte: Elaborada pelas autoras. Pesqueira, PE, Brasil (2017). Legenda: CD - Código do artigo 
Quadro 02 - Distribuição dos artigos levantados segundo código do artigo, base de dados de onde foi extraído, nome do periódico, região de realização da pesquisa e ano de publicação do artigo.

\begin{tabular}{|ccccc|}
\hline CD & Base de Dados & Periódico & RP & AP \\
\hline $\boldsymbol{A 1}$ & $\begin{array}{c}\text { Portal Regional da Biblioteca } \\
\text { Virtual em Saúde }\end{array}$ & $\begin{array}{c}\text { Revista da Rede de } \\
\text { Enfermagem do Nordeste }\end{array}$ & Sul & 2013 \\
\hline $\boldsymbol{A} \mathbf{2}$ & $\begin{array}{c}\text { Portal Regional da Biblioteca } \\
\text { Virtual em Saúde }\end{array}$ & $\begin{array}{c}\text { Revista Latino-am } \\
\text { Enfermagem }\end{array}$ & Sudeste & 2009 \\
\hline $\boldsymbol{A 3}$ & $\begin{array}{c}\text { Portal Regional da Biblioteca } \\
\text { Virtual em Saúde }\end{array}$ & $\begin{array}{c}\text { Persp. Online: biol. \& } \\
\text { saúde }\end{array}$ & Sudeste & 2015 \\
\hline $\boldsymbol{A 4}$ & $\begin{array}{c}\text { Portal Regional da Biblioteca } \\
\text { Virtual em Saúde }\end{array}$ & $\begin{array}{c}\text { Revista Geriatria \& } \\
\text { Gerontologia }\end{array}$ & Sudeste & 2013 \\
\hline
\end{tabular}

Fonte: Elaborado pelas autoras. Pesqueira, PE, Brasil (2017).

\section{Considerações Finais}

A biblioterapia ainda é um assunto escasso em estudos, artigos e ementas de graduações em saúde. É uma prática terapêutica pouco aplicada em âmbito hospitalar, e quando é feita, em sua maioria, é voltada para o público infantil, muito raramente abordada com pacientes idosos.

Isso nos dá um panorama da pouca divulgação de terapêuticas leves e que visam humanizar o cuidado. Se houvesse ênfase nestas terapêuticas poderia, sem dúvida, facilitar o trabalho dos profissionais de saúde, auxiliar o idoso em seu processo de hospitalização e facilitar a comunicação nas esferas: instituição, familiares/cuidadores, idoso.

Indubitavelmente, os elementos facilitadores para uma assistência integral precisam ser apresentados aos profissionais de saúde, lhes dando entendimento para lançar mãos de práticas que possa minimizar os danos causados por um processo de internação.

O paciente que recebe uma assistência integral, holística e humanizada sai do ambiente hospitalar com uma visão diferenciada sobre esse ciclo comum da vida, que é o adoecimento. Além de diminuir o estresse causado pelas diversas quebras de rotina vivenciadas durante o processo de hospitalização e fortalecer vínculos através do contato, comunicação e carinho.

O presente estudo evidenciou como a bilioterapia pode agir com relação a sentimentos catárticos, provocando insights nos pacientes, ademais, além de possível momento de 
relaxamento, onde sua mente deriva por outros lugares, tirando de foco a patologia e as limitações que o acometem, essa capacidade de unir fatores físicos aos psíquicos (mentais) e lúdicos, amparando ambos poderia ser amplamente difundida e vivenciada nos ambientes hospitalares com o público idoso.

\section{Referências}

ALMEIDA, Geyse Maria. A Leitura como Tratamento: diversas aplicações da biblioterapia. XIV Encontro Regional de Estudantes de Biblioteconomia, Documentação, Ciência da Informação e Gestão da informação. Universidade Federal do Amazonas. 2011.

BENEDETTI, Luciane Berto. Biblioterapia para pacientes adultos internados em uma unidade hospitalar: uma proposta de humanização. 2008. 33 f. Projeto de Pesquisa (Especialização) - Curso de Especialização em Informação Científica e Tecnológica em Saúde, Fundação Oswaldo Cruz, Porto Alegre, 2008.

BIREME (Brasil) Biblioteca Virtual em Saúde - Tutorial de Pesquisa Bibliográfica. / São Paulo: 2009. 24 p. Disponível em: http://bvsms.saude.gov.br/bvs/publicacoes/apostila.pdf . Acesso em: 12 jul. 2017.

CASTRO, Vivian Carla de; et al. Perfil de internações hospitalares de idosos no âmbito do sistema único de saúde. Revista da Rede de Enfermagem do Nordeste. 14(4):791-800. 2013.

CERIBELLI, Carina; et al. A mediação de leitura como recurso de comunicação com crianças hospitalizadas. Rev Latino-am Enfermagem. São Paulo. 17(1). 2009.

CROSSETTI, M. G. O. Revisão integrativa de pesquisa na enfermagem: o rigor científico que lhe é exigido. Revista gaúcha de enfermagem, v. 33, n. 2, p. 8-13, 2012.

FONSECA, A; GEOVANINI, F. O uso da literatura infantil na UTI: um convite à reflexão e à discussão para os profissionais sobre a morte e o morrer [Editorial]. São Paulo (SP). Revista Geriatria \& Gerontologia. 2013; 7(4): 322-326.

JERÔNIMO, Viviane; et al. Biblioterapia na melhor idade. Revista ACB: Biblioteconomia em Santa Catarina, Florianópolis, v.17, n.2, p.460-471. 2012.

MELO, Vanessa Martins de. Mediação de leitura: a biblioterapia como fator para inclusão social de idosos residentes em ILPIs. 95f. Monografia (Especialização) - Curso de Bacharelado em Biblioteconomia, Universidade Federal do Rio Grande do Sul, Porto Alegre, 2013. 
RABELLO, Janei de Souza. ZAGONEL, Ivete Palmira Sanson. MAFTUM, Mariluzi Alves. O cuidado de enfermagem ao idoso: uma reflexão segundo a teoria transcultural de Leininger. Rev. RENE. Fortaleza (CE). 8(3). 117-125. 2007.

SILVA, A.P.S.; SOUZA, B.O.R.; ANDRADE, E.R. Enfermagem e psicologia: parceria na arte do cuidar. Persp. Online: biol. \& e saúde. Campos dos Goytacazes. 18(5). 61-62. 2015.

SILVA, Candida da Silva Candida; et al. A Biblioterapia e a Intervenção Bibliotecária. XXV Congresso Brasileiro de Biblioteconomia, Documentação e Ciência da informação. Anais (v.25). Florianópolis, SC. 2013.

SOARES, CB; HOGA, LAK; PEDUZZI, M; Sangaleti, C; YONEKURA T; SILVA, DRAD. Revisão integrativa: conceitos e métodos utilizados em enfermagem. São Paulo (SP). Rev Esc Enferm USP/2014; 48(2): 335-45.

SOUZA, M. T. D. S; SILVA, M. D. D. S; CARVALHO, R. D. C. O. Revisão Integrativa: O que é e como fazer? Einstein (São Paulo) vol.8 no.1 São Paulo Jan/Mar. 2010.

VALENCIA, Maria Cristina Palhares. MAGALHÃES, Michelle Cristina. Biblioterapia: Síntese das modalidades terapêuticas utilizadas pelo profissional. Biblos: Revista do Instituto de Ciências Humanas e da Informação. Rio Grande (RS). 29 (1). 5-27. 2015.

\section{Como citar este artigo (Formato ABNT):}

BERNARDO, Juliana Maria S.; LEITE-SALGUEIRO , Claudia Daniele B.; SILVA, Paula Layse da; LOPES, Jaqueline C.; BEZERRA SOBRINHO, Aline. Reflexões sobre o Cuidado ao Idoso Hospitalizado através da Biblioterapia: Um Método Terapêutico Humanizado nos Hospitais. Id on Line Revista Multidisciplinar e de Psicologia, 2018, vol.12, n.40, p. 1037-1050. ISSN: 1981-1179.

Recebido: 06.04.2018

Aceito: 28.05 .2018 